\title{
Seventh World Congress on Emergency and Disaster Medicine Scientific Program
}

\author{
Monday, May 13, 1991 \\ 0800-1630
}

\section{$\mathbf{P}(1)(35.0) \times x \times 8$.}

Medical Evacuation of Casualties from Missile Attacks

on Israel during the Gulf War, January-February 1991

Adler J, Shemer J

Civil Defence Headquarters and Israel Defence Forces,

Medical Corps Headquarters, Tel Aviv, Israel

\section{$P(2)(33.0) \times x \times 55$.}

Maritime Radio-Medical Service: The First Ten Years

Anantharaman V, Lim SK

Accident and Emergency Department,

Singapore General Hospital, Singapore

$P(3)(10.0) 26$.

Experience of Traumatic Tracheal Disruption

Asai $Y$, Imaizumi H, Mitani M, Tomoyori T, Kobayashi K, Kaneko M

Sapporo Medical College and Hospital, Sapporo, Japan

Introduction: Tracheal disruption is very rare and formerly a lethal disease. Two cases of traumatic tracheal disruption were operated successfully.

Case 1: A 48-year-old truck driver, whose neck was jammed in a door at the time of a traffic accident, complained of severe dyspnea. Endotracheal tube insertion failed because of bleeding. Emergency tracheostomy was carried out at the lower trachea. On admission, massive subcutaneous emphysema was noted at the neck and anterior chest. Tracheal disruption was confirmed on lateral X-P, computed tomography, and fiberoscopy. End to end anastomosis of trachea were performed. Patient is doing well six months after surgery.

Case 2: A 36-year-old male who was hanged by a chain while driving a motorcycle. He complained of dyspnea. Marked subcutaneous emphysema at the neck and paradoxical movement of the trachea were noted. Tracheal disruption was confirmed on X-P and computed tomography. emergency end to end anastomosis of trachea and T-tube insertion were performed. $\mathrm{Pa}$ tient is doing well four months after surgery.

Conclusion: Report of complete tracheal disruption is very rare. Lateral neck X-P, computed tomography, and fiberoscopy were very useful in making the diagnosis of this condition. Emergency surgery was necessary and good results were obtained.

\section{$P(4)(25.0) \times x x 66$.}

Current Trends in the Management

of Oral and Maxillo-facial Trauma

Baig $M A$

Ministry of Defence and Aviation, Riyadh Al Kharj Hospital

Programme, Riyahd, Kingdom of Saudi Arabia

\author{
Monday \\ 0800-1630
}

$\mathbf{P}(5)(14.3) \times x 166$

Correlation between the Energy Metabolism

of Erythrocytes and the Disorders of Heart Rhythm

Banas UK, Sushchenko IT, Kanyuk JI, Gurenko ES

Medical Institute, L'viv, West Ukraine, USSR

Introduction: This paper reports a study of the changes in heart rhythm and the energy metabolism of erythrocytes in $131 \mathrm{pa}-$ tients age 34 to 50 years following heart mitral valve replacement surgery.

Methods: During the first 14 days following mitral valve replacement surgery, blood cells were taken from the patients and cultured. The concentrations of 2,3-diphosphoglycerate (2,3-DPG), inorganic phosphate ( $\mathrm{Pi}$ ) (after Luganiva ES, 1975), glutathione oxidative (GSSG) and reductive (GSSH) modified according to Wudvort G, 1972, and lactic (LA) and pyruvic (PA) acid were monitored (after Bergmeyer 1965). In addition, assays to determine the concentrations of $\mathrm{K}^{+}, \mathrm{Na}^{+}$, and glucose, and the $\mathrm{pH}$ of red blood cells were performed. Central and peripheral hemodynamics and electrocardiograms also were measured during the study period.

Results: Subjects included 43 patients who had tachycardia associated with atrial fibrillation. Improvement in their clinical conditions was marked by an abatement of the deficit between the heart rate and pulse wave a mean of $16.8 \pm 4.8$ strokes/ minute (min) $(\mathrm{p}<.02)$, lowering of heart rate and systolic arterial pressure to mean values of $92.8 \pm 4.1$ strokes/min and $126.7 \pm 2.5 \mathrm{~mm}$ $\mathrm{Hg}(\mathrm{p}<.01)$ respectively. Accordingly, in erythrocytes there was a correlation of LA to PA which increased to $16.2 \pm 5.9(\mathrm{p}<.001)$, and $2,3-\mathrm{DPG} / \mathrm{Pi}$ increased to $7.5 \pm 2.2(\mathrm{p}<.001)$. There was a corresponding increase in the mean concentration of LA to $7.22 \pm$ 0.01 ( $\mathrm{p}<.001$ ). Mean $\mathrm{pH}$ increased to $7.103 \pm 0.019$ ( $\mathrm{p}<.001)$, was associated with an increase in mean $\mathrm{K}^{+}$content to $82.61 \pm 1.96$ $\mathrm{mMol} / \mathrm{L}(\mathrm{p}<.001)$, and the GSSG/GSSH decreased to $0.23 \pm 0.17$ $(p<.002)$, which allowed maintenance of the activation of the pentose-phosphate cycle against a background of considerable augmentation of glycolysis.

Bigeminal rhythm was detected in 11 patients, and a marked increase in mean LA in the erythrocytes by $2.38 \pm 0.32 \mathrm{mMol} / \mathrm{L}$ $(\mathrm{p}<.002)$, and a sharp increase in the LA/PA to $300.7 \pm 40.2$ $(\mathrm{p}<.001)$ was associated with this rhythm.

The 77 patients who experienced sinus node tachycardia when their heart rate decreased by a mean value of $20.7 \pm 1.1$ strokes/min $(\mathrm{p}<001)$, showed a concentration of $\mathrm{K}^{+}$that increased from a mean level of $68.30 \pm 1.95$ to $79.35 \pm 1.86 \mathrm{mMol} / \mathrm{L}$ $(\mathrm{p}<.001)$, and the mean content of LA decreased to $2.01 \pm 0.16$ $\mathrm{mMol} / \mathrm{L}(\mathrm{p}<.02)$.

Conclusions: The correlation between the energy state of erythrocytes and the clinical variations in the disorders of heart rhythm is an important diagnostic test of the treatment used and of the effectiveness of intensive care treatments.

Vol.6, No.2 
Monday

0800-1630

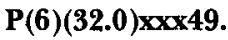

Medical Rescue Car: An Integrated Emergency System

Barbero M, Bermano F, Esposito S, Robba $F$

Hospital S. Paolo, Savona, Italy

$\mathbf{P}(7)(34.2) \times x \times 50$.

A National Program for Medical Preparedness

Bosner LV, Jordan LS

Emergency Preparedness Committee, D.C. Partners of Brasilia,

Washington, D.C., USA

$\mathbf{P}(\mathbf{8})(13.0) \times x \times 10$.

Using Regression Analysis to Predict Emergency

Patient Volume at the Indianapolis 500 Mile Race

Bowdish GE, Cordell WH, Bock HC, Vukov LF

Mayo Medical School, Rochester Minnesota, USA

Emergency Medicine and Trauma Center, Methodist Hospital of

Indiana, Indianapolis, Indiana, USA

$\mathbf{P}(9)(34.1) \times x \times 9$.

The Plainfield, Illinois, Tornado Disaster

Brenner $S A$, Nöi $E$

Centers for Disease Control, Atlanta, Georgia, USA

On 28 August 1990, a tornado struck Will County, Illinois, killing 28 persons and causing over [US] \$2-million in damage to property. There had been no tornado warning issued.

The Centers for Disease Control conducted an investigation to assess risk factors for death and injury, utilizing medical records, telephone interviews, coroners reports, and Red Cross data. In order to ascertain the contribution of different types of buildings to the risk of death and injury, information on building construction methods and materials was obtained from tax assessor records.

The majority of those fatally injured died instantaneously, although five persons initially were hospitalized. One person died eight weeks after suffering impact-related injuries. Many of those killed were younger than 20 years old. Due to the lack of prior warning, many persons were unable to get to a safe tornado shelter. Location in the basement of a house proved to be the safest place during the tornado; houses with only one story proved to be more protective that those with more floors. In the period after the tornado, over $60 \%$ (221 patients) of those presenting to emergency rooms went to one hospital.

These findings emphasize the importance of early warning systems in tornado-prone areas, construction of tornado-resistant shelters, public knowledge of what to do when a tornado hits, and the need for continuing surveillance for at least 8-12 weeks in the post-disaster period. Pre-disaster triage planning may not predict the patterns of admissions to emergency departments, since many patients arrive by means other than ambulance. Hospital personnel should be involved in community disaster planning
Monday

0800-1630

\author{
$\mathbf{P}(10)(32.1) \times \times x 60$. \\ Health Organizations in Mass Manifestations [Gatherings] \\ Castellini PL, Misley A, Vicini P \\ Policlinico Modena, Associazione Volontariato, Vignola, Italia
}

P(11)(35.13)xxx118.

Medical Supplies and Equipment for Disasters

Ciancamerla $G$, Pagliaro $F$

Italian Red Cross Volunteers, Aosta Valley Committee, Aosta, Italy

$\mathrm{P}(12)(34.18) \times \times 158$.

Triage in Mass Casualty Situations

Debacker $M$, Delooz $H$, Bellanger $C$, Winnen $G$

Medical Service, Belgian Armed Forces; Catholic University of Leuven;

Medical Service, Belgian Armed Forces. Brussels Belgium

$P(13)(35.4) \times x \times 56$.

The Current Approach to Accidents

and Disasters in the Netherlands

De Boer J

Civil Defence and Peacetime Calamities, Ministry of Welfare, Health, and Culture, Rijswijk, Netherlands

P(14)(34.4) $\times x \times 67$.

The PUSH System: Event-Triggered Medical

Response to Catastrophic Disasters

Freeman $C$

California EMS Authority, Sacramento, California, USA

$P(15)(40.0) \times x x 5$.

Emergency Medicine at Sea

Heller $I L$

Section of Emergency Medicine, Virginia Mason Clinic, Seattle,

Washington, USA 
Monday

0800-1630

$\mathbf{P ( 1 6 ) ( 3 8 . 1 ) \times x x 1 1 . ~}$

Medical Command Errors in an Urban ALS System

Holliman CJ, Wuerz RC, Meador SA

Division of Emergency Medicine, The Milton S. Hershey Medical

Center, The Pennsylvania State University, Hershey, Pennsylvania, USA

Objective: The aim of this study was to identify the most common types of errors made by on-line medical command so as to be able to assist in focusing educational efforts for command physicians.

Design: Retrospective survey of prehospital ALS trip sheets.

Setting: An urban ALS paramedic service with on-line physician medical command rotating on a monthly basis between three hospital.

Participants: All ALS runs from September 1988 through December 1990 were reviewed as part of an ongoing quality assurance $(Q / A)$ program. Cases were identified as deviating from regional EMS protocols or generally accepted standards of care as judged by agreement of three physician reviewers. Cases were excluded when all three reviewers did not agree that the command rendered was inappropriate.

Interventions: Command errors were identified from the prehospital ALS run sheets and were categorized.

Results: A total of 199 command errors in 166 cases were identified from 3,939 runs (5.2\% of all runs). Seven types of errors accounted for $84 \%$ of the total errors, with the most common error $(34 \%)$ being failure to address the possibility of hypoglycemia in patients with an altered level of consciousness. The error rate decreased from $8 \%$ to $2 \%$ of total runs over the period of study. Conclusions: To reduce the medical command error rate, physician education should be directed at the seven problem areas identified. Ongoing $Q / A$ review of medical command may result in decreasing the command error rate.

\section{$P(17)(29.6) \times x x 120$.}

Long-Term Medical Stabilization of Hemodynamics in Patients After Emergencies and Energy Metabolism of Erythrocytes. Horbai $O$, Banas' $U$, Danylenko $M$, Gurenko E.

L'vì Medical Institute, West Ukraine, USSR

Tactics of treatment of patients with unstable hemodynamics with catecholamines after different emergencies were studied on a group of patients after they had undergone a heart operation. The purpose of this study was to investigate the use of dopamine in achieving hemodynamics stabilization.

Methods: The content of 2,3 diphosphoglycerate (2,3-DPG) as well as the content of inorganic phosphate (Pi), oxidative (GSSG) and reductive (GSSH) glutathione, lactic (LA) and pyruvic (PA) acids, $\mathrm{K}^{+}, \mathrm{Na}^{+}, \mathrm{pH}$ and glucose of red blood cells (erythrocytes) were measured before an infusion of dopamine during the 1st, 3-4th, 6-7th, and 12-14th days.

Conclusions: The use of high doses of dopamine over the longterm oppresses glycolysis and the pentose-phosphative pathway of metabolism. This depresses the energy state of erythrocytes: metabolism is worsened in cells where glycolysis is the basis of their energy production. Along with the definite changes of erythrocyte metabolism, definite disorders of heart rhythm were observed. Therefore, high doses of dopamine can be used only momentarily and only in accordance with erythrocyte metabolism. It is necessary to conduct more research directed at correction of disorders of a heart rhythm.

Reference: Theses of the 4th All-Union Science Conference, Prof. Mycaelyan (ed), Yerevan, USSR 1989;84.
Monday

0800-1630

$P(18)(6.5) \times \times 72$.

Carbon Monoxide Poisoning in a Gas Explosion

Ihara $K$, Goami $K$, Taniguchi M, Morimoto T, Hara N, Kimura $M$, Nozaki K, Oomura I

Kure National Hospital, Kure City, Hiroshima Prefecture, Japan

$P(19)(7.1) \times x x 28$.

The Acute Effects of Nicardipine on Hemodynamics and Electroencephalogram Abnormality

Ikeda K, Ueda K, Neya K, Hamasaki Y, Takei K, Hoshino Y, Asakawa $K$, Ishiguro $T$

ICU, Ohmiya Seki-Juji Hospital, Yono, Saitama, Japan

Nicardipine (NC), a new calcium antagonist, has a potent peripheral and cerebral vasodilating activity and is used for treatment of hypertensive emergencies. The effects of NC on hemodynamics and electroencephalogram (EEG) in patients (pt) after open heart surgery were evaluated, because hemodynamic instabilities, such as severe hypotension, increased systemic vascular resistance, low cardiac output, and EEG abnormalities often follow open heart surgery.

Methods: Hemodynamic Studies-In nine patients following cardiac surgery, NC was administered intravenously (IV) and hemodynamics were measured over a 3-hour $(\mathrm{h})$ period. The dosage of NC was adjusted by constant IV infusion so to reduce mean arterial wedge. EEG Study:-Fifteen pts were studied. In eight pts (NC group), NC was administered after surgery, and seven pts did not receive NC (non-NC group). Pre- and postoperative EEGs were evaluated by Fourier analysis. In each pt, the summation of the power spectrum of delta (D) and alpha (A) waves was obtained, and D/A was calculated.

Results: Hemodynamic Effects-With NC treatment, the mean arterial blood pressure (MAP) was reduced consistently from $88.0 \pm 12.7$ to $70.7 \pm 5.5 \mathrm{mmHg}(\mathrm{p}<.02)$. Systemic vascular resistance index (SVRI) decreased from $1899 \pm 546$ to $1474 \pm 303$ dyne sec $\mathrm{cm}^{-5} \mathrm{~m}^{2}$ ( $\left.\mathrm{p}<.02\right)$, and stroke volume index increased by $14 \%$. EEG Effects-After surgery, in the non-NC group, mean $D$ increased by $59.1 \%$ and mean $A$ decreased by $32.0 \%$, with a mean increase in D/A of $164.6 \%$. In NC group, the increase of $\mathrm{D}$ and decrease of $\mathrm{A}$ were less pronounced post-operatively, and the mean D/A increased only $31.2 \%$.

Conclusions: The blood pressure reducing effect of NC was demonstrated by SVRI reduction and SVI increment, which is compatible with hemodynamic improvement in $\mathrm{pt}$ after open heart surgery. It also suggests that NC has efficacy in the improvement of cerebral circulation, because post-operative changes of delta and alpha waves became less pronounced following NC administration. 
Monday

$0800-1630$

$\mathbf{P}(20)(12.0) \times x \times 29$.

Small Intestine Rupture Due to Blunt, Abdominal Trauma:

The Clinical Evaluation of Computed Tomography

Imaki S, Yamanaka I, Ohhama N, Ogawa T

Introduction: Physical examination often is unreliable in the evaluation of victims who have suffered blunt, abdominal trauma. Therefore, diagnosis is very difficult, and delays in diagnosis and treatment worsens morbidity and mortality. In regard to hepatic, splenic, and renal injuries, computed tomography (CT) is very useful in the early diagnosis of blunt, abdominal trauma. However, the utility of CT in small intestinal and mesentery injuries at an early stage is controversial.

Methods: The utility of CT in the rupture of the small intestine was evaluated by comparison of physical findings, plain abdominal films, and operative findings. Pre-operative CT of $11 \mathrm{pa}-$ tients with surgically proven small intestine rupture were evaluated retrospectively.

Results: In the CT on admission, four cases showed a small amount of obvious free air with intra-abdominal hemorrhage, three cases showed normal findings, and six cases underwent immediate laparotomy. The others had a follow-up CT study within 24 hours; while the four cases that showed free air or increasing hemorrhage underwent laparotomy. In only one case, a small intestinal rupture could not be detected by CT. Plain abdominal films on admission failed to reveal rupture in any of the cases.

Conclusion: The study shows CT to be very useful and significant to the early diagnosis of small intestinal rupture due to blunt, abdominal trauma.

\section{$P(21)(16.6) \times x x 90$.}

\section{Acute Poisoning of Phenylpropanolamine}

Preparations in Japan

Isomura CI

Japan Poison Information Center, Osaka, Japan

More than 90 over-the-counter (OTC) phenylpropanolamine (PPA) are available as nasal decongestants or bronchodilator in Japan. The highest dose of PPA in one OTC tablet is $70 \mathrm{mg}$, which is very close to its toxic dose (100 $\mathrm{mg}$ in adults). Accordingly, PPA poisoning may occur by ingestion only of 1.5 tablets. Among 99 inquiries about PPA ingestions received by the Japan Poison Information Center (JPIC) in 1989, 92 were cases were due to the ingestion of OTC drugs, of which 89 were cases of accidental ingestion. In 72 of those cases, the dose ingested was higher than the toxic dose. The main symptoms of PPA-poisoned patients were palpitation, tachycardia, nausea, and hypertension. Fortunately, no serious symptoms were noted. However, PPA needs more attention paid to its use as the OTC drugs contain potentially dangerous ingredients.

\section{$P(22)(8.4) \times x \times 159$.}

Case Presentation

Juul $O$, Compton $M$, Selig $M$, Harlow D

Ambulance Education Centre, Royal North Shore Hospital,

St. Leonards, Australia
Monday

$0800-1630$

$\mathbf{P ( 2 3 ) ( 1 6 . 1 ) \times x 9 2 . ~}$

Elevated Colloid Osmotic Pressure of Bronchial Fluid in Subarachnoid Hemorrhage Patients with Pulmonary Edema Kajita MI, Kurosawa T, Sheng HM, Wakabayashi Y, Hirata M, Kikuno T, Kitahara T, Soma K, Ohwada T

Department of Emergency and Critical Care Medicine, Kitasato University School of Medicine, Sagamihara, Japan

The pulmonary edema which occurs following central nervous system (CNS) diseases often is called "neurogenic pulmonary edema" (NPE). The proposed mechanism for the development of NPE may be a centrally mediated massive neural discharge following initial insult to the CNS.

Methods: To clarify the mechanisms of NPE in patients with a subarachnoid hemorrhage (SAH), bronchial fluid and serum samples were studied from eight patients who suffered from aneurysmal SAH with severe pulmonary edema. The bronchial fluid and serum samples were collected simultaneously. Colloid osmotic pressure (COP) was measured with a transducer-membrane system (Weil Oncometer, Instrumentation Laboratories, Lexington, Mass., USA).

Results: Of the eight SAH patients, seven were placed in Fisher group 3 and one was placed in group 4 on the basis of a brain CT. The COP of bronchial fluid and serum samples were $16.7 \pm$ 4.1 and $18.2 \pm 3.2 \mathrm{mmHg}$ respectively. The COP ratio of bronchial fluid to serum was $0.94 \pm 0.26$, ranging from 0.64 to 1.45 .

Conclusion: In the present study, one of the mechanisms of NPE in SAH was thought to be increased pulmonary capillary endothelial permeability which may play a role to varying degrees in the development of pulmonary edema.

\section{P(24)(34.6) $\times x 86$.}

\section{Disaster Planning in Bangladesh}

Karim $N$

Disaster Preparedness Centre of Bangladesh, Dhaka, Bangladesh

This paper shall deal with the process of disaster planning in Bangladesh. Disasters can occur at any time, in any place, in all types of weather, and may owe as much to the circumstances as to the scale of the event. With the exception of the man-made disaster of war, the worst disasters follow natural events. Planning should start with clear recognition of the fact that, however a disaster is defined, arrangements must be made to notify those affected by the disaster.

The state of disaster planning varies due to time and situation. The unprecedented flood of 1988, which occurred throughout Bangladesh, had stimulated fresh planning and work. All areas had plans, but even the best of them can never be perfect. One of the best ways to improve plans is to learn from the experiences of other people. This is the rationale for the second part of this paper in which a range of different events that have occurred in Bangladesh in the recent past are described.

$\mathbf{P}(25)(26.0) \times x \times 30$.

External Fixation [Immobilization]

in the Third Level Trauma Center

Kawai M, Henmi HH, Yamamoto YM, Mashiko KM, Otsuko TO

Department of Emergency and Critical Care Medicine, Nippon

Medical School, Tokyo, Japan 
Monday

0800-1630

$\mathbf{P}(26)(35.5) \times x x 17$.

Disaster Reanimatology Potentials:

Appraisal of Structured Interview Study in Armenia

Klain M, Safar P, Ricci E, Semenov V, Pretto E, Tisherman S, Abrams I

International Resuscitation Research Center (IRRC), University of

Pittsburgh, Pittsburgh, Pennsylvania, USA, and Institute for General

Reanimatology, Moscow, USSR

$\mathrm{P}(27)(8.6) \times \times \times 4$.

Recurrent Episodes of Ventricular Flutter

and Fibrillation Due to Potassium Deficiency

Liao LT, Tao $S Q$

Nephrology Department, Zhongshan Hospital, Shanghai Medical University, Shanghai, PRC

Potassium deficiency is a common cause for repetitive episodes of ventricular flutter (VF). Assessment of potassium balance has not been stressed during the time of or following cardiac resuscitation, when substantial doses of alkaloids, diuretics, and steroids have been administered.

Nine hypokalemic patients, who had some form of non-organic heart disease, developed recurrent episodes of VF and ventricular fibrillation (Vf). In five cases (56\%), 2 to 6 episodes of dysrhythmias occurred within 1 to 24 hours $(h)$. One patient suffered 31 attacks within $66 \mathrm{~h}$, and another had 33 attacks in $7 \mathrm{~h}$. Of the 59 episodes registered, 55 were VF, with the characteristics of high amplitude and frequency, no response to antiarrhythmic drugs, without spontaneous conversion to ventricular tachycardia (VT) or other cardiac rhythms, and were liable to recur. Each episode lasted for 10 seconds to two minutes. Between episodes, transient recurrent episodes of short runs of polymorphous VT, sometimes torsades de pointes, multi-focal and bizarre premature ventricular complexes-on-U phenomenon, and/or even impure QRS configurations frequently were seen and usually were specific.

Most VF responded temporarily to direct current cardioversion. Isuprel infusion proved to be successful in the urgent treatment of $\mathrm{VT}$ with prolongation of $\mathrm{Q}-\mathrm{T}(\mathrm{Q}-\mathrm{U})$ interval including torsade de pointes. Patients with hypokalemia-induced $\mathrm{VF}$ were considered to have severe potassium deficiency. Hence, large doses of potassium chloride (about $10 \mathrm{~g} / \mathrm{d}$ ) in a concentration of 0.6 to $1.0 \%$ were infused at a rate of $1-2$ grams during the first hour. Additional supplements of potassium were given until serum potassium levels were stabilized within the normal range and the hypokalemia-induced abnormalities disappeared.

Sometimes, potassium deficiency can exist in spite of normal serum levels. This could be due, in part, to metabolic acidosis resulting from recurrent episodes of cardiac arrest. Among the nine patients, two died; one from VF, and the other due to unrecognized hypokalemia.

$\mathbf{P}(\mathbf{2 8})(\mathbf{3 6 . 0}) \mathbf{x \times x 2}$.

A Computerized System for the

Management of the Traumatic Patient

Magalini S, Proietti R, Michetti $V$

Institute of Anaesthesiology and Reanimation, Università Cattolica del

Sacro Cuore, Rome, Italy
Monday

$0800-1630$

P(29)(13.1) xxx3.

Telemedicine in Disaster and Emergency Medicine

Manni $C$

Institute of Anaesthesiology and Reanimation,

Università Cattolica del Sacro Cuore, Rome, Italy

$\mathbf{P}(30)(33.3) \times x 52$.

Pulse Wave Oximetry during Ambulance Transportation

Mattila MAK, Koski EMI

Kuopio University Hospital, Kuopio, Finland

Introduction: At present, patient monitoring during emergency transportation is minimal. Only a few variables are measured at random intervals. Thus, the reports available upon arrival at the hospital contain only minimal data regarding the patient's prehospital state. Patients in critical condition need monitoring to assess their physiological condition and to determine the effects of applied therapy. Modern monitoring devices can be used in the field and can provide data to assist in the observation of patient trends. These data trends may be printed out or digitalized later for further processing.

Methods: The performance of a portable pulse oximeter (SatliteTrans, Datex/Instumentarium Co., Helsinki, Finland) was evaluated to determine any possible variability of peripheral pulse wave amplitude measured on a physician-manned ambulance. Special attention was focused on the monitoring of peripheral pulse-wave amplitude and on the reliability of the method. Monitoring was begun immediately at the scene and continued until arrival at the hospital. Values for pulse rate, oxygen saturation, and pulse wave amplitude were averaged every 10 seconds and these data were transmitted to a Personal Computer (PC) using data collection software (Lotus Measure) for further processing on a spreadsheet program (Symphony). The artifact values were selected manually and their incidence was counted. The various phases of transportation were analyzed separately.

Results: The pulse-wave amplitude reflected changes in the peripheral pulse volume. Various factors(decreased circulatory volume, pain, stress, and external temperature) can influence peripheral circulation. The recorded data evidenced a marked tendency for vasoconstriction during transportation. However, the performance of the monitor was adequate during transportation, and a low incidence of artifact values $(<4 \%)$ was observed.

Conclusion: This study indicates that this type of pulse oximeter is suitable for use in the field and during transportation. The information obtained can be used instantly for patient assessment and as a basis for therapeutic decisions. In mass casualty situations, pulse oximetry can be a valuable tool in triage decisions. The collected data trends can be used for reconstruction of the situation, for learning, and for instructional purposes. 
Monday

$0800-1630$

$\mathbf{P}(31)(23.2) \times \times \times 32$.

Significance of CT During Barbiturate

Therapy for Head Injuries

Morinaga KM, Okawara SO

Okawara Surgical Hospital, Muroran, Japan

Barbiturate therapy is used to control intracranial pressure in patients with severe head injuries. With an aim of determining the duration for barbiturate administration, brain computerized tomography (CT) was performed during barbiturate therapy, and the findings were used to study its usefulness for this purpose.

Methods: The subjects included seven patients with severe head injuries who were admitted to Okawara Surgical Hospital during the past two years. Each patient received barbiturate therapy and their clinical course was observed by brain CT.

Results: 1) Improvements of brain CT findings (degree of visualization of the periencephalic cistern and midline shaft) was found in four cases. Two cases, in which the dose of barbiturate was reduced rapidly, developed cerebral herniation and died. Of the two cases in which the barbiturate therapy was continued, one was rated as having moderate disability and the other had good recovery. 2) Delayed intra-cerebral hematoma developed during the barbiturate therapy in four cases. In two cases, the hematoma was removed during the barbiturate therapy and the outcome was good.

Conclusions: The results suggest that monitoring of delayed intra-cerebral hematoma is important during barbiturate therapy, but that improvement in findings of brain CT in the acute phase cannot be used as a yardstick for reducing the dose of barbiturates.
Monday

$0800-1630$

$\mathbf{P}(34)(35.6) \times x \times 106$

Perspectives on the Use of Extracorporeal

Detoxification in Disaster Medicine

Nechaev E, Gurevich C, Shwedov A

Medical Military Academy, Leningrad, USSR

Most catastrophes involve a large number of victims with physical trauma, burns, crush syndrome injuries, exotoxicoses, radiation, drowning, freezing, or mental trauma. Along with these physical injuries comes an increased probability for the development of infection. Intoxication [toxemia] syndrome, violation of blood rheology, and fluid, electrolyte, and protein balance play an important role in the pathogenesis of these conditions. These insults make extracorporeal detoxification necessary in order to counteract the pathogenic effects resulting from such injuries. The products are managed by means of direct and indirect detoxification, immuno-correction, and rheo-correction. Each of these processes considerably expands the possibilities for transfusion therapy.

Experience with the use of extracorporeal detoxification in 205 combat, catastrophe, and disaster victims suggests that its use promotes a positive clinical outcome $(84 \%)$ in wounded and sick patients. The operations which can be performed using this technique include: plasmapheresis $(\mathrm{PH})$, plasma-carboperfusion (PC), hemo-carboperfusion (HC), hemo-oxygenation (HO), ultrafiltration (UF), hemodialysis (HD), and such combinations as $\mathrm{PH}+\mathrm{PC}, \mathrm{PH}+\mathrm{HO}, \mathrm{HC}+\mathrm{HO}, \mathrm{UF}+\mathrm{HC}$, and $\mathrm{PH}+\mathrm{PC}+\mathrm{HO}$.

Indications used for the initiation of extracorporeal detoxification included: severe injuries of soft tissue; a high level of intoxication [toxemia], signs of the DIC syndrome, acute renal insufficiency, sub- and decompensated ischemia of extremities due to damage to large vessels, severe burns, acute poisoning, radial disease, generalized forms of acute infectious diseases, and acute mental reactions in cases resistant to traditional treatment. The need for extracorporeal detoxification was $0.5 \%$ (average) on the stage of qualified medical care, and $1.6 \%$ (average) on the stage of specialized care.

The organizational principles and facilities for extracorporeal detoxification were developed on the basis of these data. The main tasks of extracorporeal detoxification methods for use in Disaster Medicine are: specification of indications and contraindications; development of universal transportable equipment; and the availability of mass-exchanging columns. 
Monday

0800-1630

$\mathbf{P ( 3 5 ) ( 4 0 . 1 ) \times x x 3 1 . ~}$

The 15 Years of Nippon Medical School Emergency

and Critical Care Medical Center in Japan

Ninomiya N, Henmi $H$, Yamamoto $Y$, Kurokawa $K$, Mashiko $K$,

Yasuda K, Otsuka T

Nippon Medical School Emergency and Critical Care Medical Center,

qoyko, Japan

The Nippon Medical School Emergency and Critical Care Medical Center (NMSECCM) was established in April 1975. This paper presents the 15 year history of the NMSECCM Center. The Center, centrally located in Tokyo, is the city's largest emergency medical facility, and it provides treatment for the city's most severely injured patients. In its 15 -years of operation, 11,518 patients were admitted, of which 8,162 were male $(71 \%)$ and 3,356 were female $(29 \%)$. The average length of stay was 8.8 days, and 3,497 patients died $(30.4 \%)$. The patients came from the Tokyo metropolitan area, neighboring prefectures, adjacent islands, and from the Pacific Ocean. On average, more patients were admitted between October and March than between April and September, and the peak time for admissions was between 1000 hours and midnight.

The admissions have increased each year. The number of admissions for 1975 was 274 ; the highest number of admissions, 1364 , occurred in 1989. There were 4,812 surgical operations performed during the 15 years of operation. Every day, the staff makes an effort to improve the level of emergency medical service offered at Nippon Medical School Emergency and Critical Care Center.

P(36)(19.4)xx97.

Undergraduate Emergency Medical Training in Japan

Ohashi N

Tsukuba Medical Center, Ibaragi, Japan

$\mathbf{P}(37)(11.0) \times x x 27$.

Clinical Assessment of Severe Status Asthmatics

Ohhama N, Yamanaka I, Ogawa T, Nagano K, Imaki S, Akanuma K St. Marianna University School of Medicine, Yokohama City Seibu Hospital, Yokohama, Japan
Monday

$0800-1630$

S3(38)(16.3) $\times x \times 64$.

Prognostic Factors of Acute Pulmonary Edema

Omboni E, Checchini M, Minora T, Pediconi A, Pierini A, Sardella F

Medical Emergency Department, Fatebenefratelli-Oftalmico Hospital,

Milano, Italy

This study provides an analysis of the evolution of acute pulmonary edema (APE) and the prognostic factors influencing the short-term survival of 108 patients admitted with APE. Of the 108 patients, $87(80.5 \%)$ were discharged and $21(19.5 \%)$ died. Goldberger at al $^{1}$ found that patients with initial systolic blood pressure (SBP) $\geq 160 \mathrm{mmHg}$ showed longer survival compared with patients with SBP $<160 \mathrm{mmHg}$. No other significant prognostic factors were identified at the time of admission. Patients were classified into two groups-survived and deceasedand the differences between the two groups were analyzed with chi-square tests with Yates correction and with One-Way ANOVA.

Results: A better outcome was achieved in: a) patients with a previous history of cardiac failure $(\mathrm{p}=.02)$; b) younger patients (mean age $75.6 \pm 9.0$ years in the surviving group and $83.4 \pm 8.2$ years in the deceased group); c) patients with higher SBP $(173.2 \pm 37.7 \mathrm{mmHg}$ and $134.6 \pm 47.0 \mathrm{mmHg}$ respectively); d) patients with higher plasma levels of bicarbonate $(20.1 \pm 5.2$ $\mathrm{mEq} / \mathrm{L}$ and $16.2 \pm 5.6 \mathrm{mEq} / \mathrm{L}$ respectively) and base deficit $(-5.4 \pm 5.8$ and $-10.6 \pm 6.8 \mathrm{mEq} / \mathrm{L}$ respectively).

1.Goldberger IJ, et al: Arch Intern Med 1990;146:489.

$\mathbf{P}(39)(32.2) \times x 151$.

What Skills Are Necessary: Prehospital?

Oxer $H$

St. John Ambulance, Belmont, Western Australia

The range of prehospital skills varies from first aid to paramedics carrying up to 60 different medications, and having a wide range of manual skills. Surprisingly, there is little information on which of these skills and drugs affect patient outcome.

The uncritical transfer of many in-hospital techniques to the out-of-hospital setting has resulted in expensive services. From time-to-time, the occasional "voice in the wilderness" has queried whether much actually is needed in the prehospital setting. There is ample evidence that early prehospital defibrillation saves lives, though the overall numbers are small. There is little evidence that any other prehospital cardiac intervention actually affects outcome to any significant degree.

An important review article by Michael Schuster (1989) assessed the evidence for effectiveness of pharmacologic intervention in prehospital care. He was unable to document that any medication given by the pre-hospital care provider was beneficial, or could not be delayed safely until arrival at hospital. Champion and others have promoted "Scoop and Run" as the most effective management of serious trauma. Potter, Goldstein et al carried out a controlled trial of pre-hospital advanced life support in trauma in Australia, and when 500 cases handled by paramedics were compared with over 500 who receive BLS care only, were unable to show any benefit of paramedic intervention that affected outcome. Paramedic journals are full of esoteric articles concerning detailed analysis of electrocardiographic wave-forms, but are very light on how that affects prehospital management and patient outcome. The more costeffective way to manage prehospital care may be to train staff only in those skills which have been shown to improve patient condition or outcome. 
Monday

0800-1630

$\mathbf{P ( 4 0 ) ( 2 3 . 5 ) \times x 1 7 0 . ~}$

Intra-operative Use of Ultrasonography

during Craniotomy of Head Injury

Osaki T, Kusunose M, Tanabe H, Osaka-fu Mishima Critical Care

Medical Center, Takatsuki City, Osaka, Japan

$P(41)(5.2) \times x 138$.

Evaluation of Propranolol Efficacy in Myocardial Infarction Complicated by Heart Failure and Ventricular Arrhythmias

Parkhomenko A ,Kiev Medical Institute, Kiet, Ukraine, USSR

Introduction: To evaluate the actions of propranolol (PR) in acute myocardial infarction (AMI) with heart failure (HF) and ventricular tachycardia/fibrillation (VT/VF) during the 1st day of an AMI, 74 patients (pts) were observed.

Methods: Hemodynamics, pulmonary gas exchange, ECG mapping, and Holter monitoring were studied. Programmed ventricular stimulation (PVS) with 1-2-3 extra stimuli in right ventricular apex also was used. 31 patients received conventional treatment (Group I), and 43 patients were treated additionally with PR (Group II).

Results: After PR administration, complex arrhythmias were registered more rarely. Left ventricular filling pressure, stroke volume, and oxygen delivery were unchanged, but patients had a decrease in blood pressure, heart rate, and an elevation in tissue oxygen consumption. During in-hospital follow-up, suppression of Holter detected ventricular arrhythmias, improvement in heart function, and more rapid termination of HF were found in the Group II. Also, the reduction in sustained VT-VF inducibility at 7-26 day of MI in PVS were observed in PR treated patients (from 22.5 to $9.3 \%$ )

Conclusions: Thus, PR administration is an effective method for reduction of heart electrical instability in AMI patients with $\mathrm{HF}$ and life-threatening ventricular arrhythmias (VT/VF).

\section{$\mathrm{P}(42)(9.5) \times x 146$.}

"Lactoproteinum" Influences Upon Middle Mass Molecule (MMM) Levels in Dog's Blood during Hemorrhagic Shock Pavlousky M, Oborin A, Zubachik R, Mindjuk M

Department of Surgery, State Medical Institute, Lvov, USSR

Ongoing severe shock is accompanied, as a rule, by the beginning of endotoxicosis (ET), in which a considerable role is played by oligopeptides of middle molecules, so called MMM. The MMM impair cell functions. The "Lactoproteinum" (LP) influence upon MMM level in blood taken from the v. cava caudalis of seven dogs that underwent irreversible hemorrhagic shock, was studied by means of spectrophotometrical method in the wave length of $254 \mathrm{~nm}$.

Lactoproteinum is a $\mathbf{5 \%}$ albumin solution containing glucose, $\mathrm{K}+, \mathrm{Na}+\mathrm{Ca} 2+$, sodium lactate, and bicarbonate. It was infused rapidly into the $\mathrm{v}$. femoralis at a rate of $20 \mathrm{ml} / \mathrm{min}$ after $7.4+1.5$ hrs. of hemorrhage during which the arterial blood pressure was maintained at levels of $30 \mathrm{mmHg}$.

It was established that irreversible hemorrhagic shock increases the MMM level by 35\% ( $p<.0001)$. Also, after 1 hour of LP infusion, MMM levels were not different from the initial levels ( $>.5$ ). These results can be explained by the ability of albumin for oligopeptide binding. But, after 24 and 48 hours, the MMM levels increased in $17 \%(\mathrm{p}<.1)$ and $27 \%(\mathrm{p}<.01)$ respectively as a result mainly of liver detoxification function disorders. All the dogs survived.
Monday

$0800-1630$

The obtained data were to establish the necessity to use L.P in the complex ET's therapy .

P(43)(35.7) $x \times x 18$.

Disaster Reanimatology Potentials:

A Structured Interview Study in Armenia III:

Results, Conclusions, and Recommendations

Pretto E, Ricci E, Klain M, Safar P, Comfort L, Semenov V, Abrams J, Tisherman S, Crippen D, Angus D, and other member of the Disaster Reanimatology Study Group

International Resuscitation Research Center (IRRC), and Schools of Medicine, Public Health, Public and Intermational Affairs, and Engineering, University of Pittsburgh, Pittsburgh, Pennsylvania, and the USSR Academy of Medical Sciences, Moscow, USSR

Introduction and Methods: An interview study of the 1988 Armenia (USSR) earthquake was conducted to examine medical care delivery and potentials for life-supporting first aid (LSFA) and advanced trauma life support (ATLS) within the first 24 hours. The methods employed and preliminary results have been reported. 1

Final Results: 1) Medical teams arrived within two hours but had inadequate extrication equipment and resuscitation supplies; 2) There was limited use of LSFA and ATLS in the field; 3) In the first few hours, medical respondents (MRs) mainly dealt with victims with multiple injuries and shock, crush injury, fractures, external bleeding, head injury, and airway problems; 4) Some MRs observed victims who died slowly who might have been saved by earlier ATLS; 5) The only field operations performed were amputations; 6) The international response was minimal before Day 3, which was too late for the successful application of LSFA and ATLS; 7) Most lay respondents (LRs) had been trapped under rubble $(92 \%)$ for a mean of 21 hours, and most were extricated by friends or family (59\%); and 8 ) Almost half of the LRs saw or heard victims under rubble die slowly without medical care.

Conclusion: Civilians administering LSFA would save lives. Extrication and ATIS skills might enhance local response team effectiveness.

Recommendations: Conduct further research into: 1) Basic extrication (BE) and LSFA training for high-risk populations; 2) Integration of medical and rescue responses; and 3) The importance of time in the efficacy of the international response.

1. Klain M, et al: Disaster reanimatology potentials: A structured in t e r view study in Armenia: I. Methodology and preliminary results. Prehos. pital and Disaster Medicine 1989;4:135-152.

P(44)(35.14) $\times x 124$.

Prototype: The Emergency Department Command System

Rega P, McConnaughy D, Mack D

The Toledo Hospital, Toledo, Ohio, USA

$\mathrm{P}(45)(29-4) \times x \times 179$

Acupuncture-Based General Anesthesia

Rosenberger FM, Theobaud $C$

International Pain Relief Association, Graz, Austria

$\mathbf{P ( 4 6 ) ( 3 4 . 9 ) \times x x 1 9 .}$

Peace Medicine and Acute Combat Casualty Care

Safar P, Stremple I

International Resuscitation Research Center (IRRC), and

Departments of Anesthesiology and Surgery, University of Pittsburgh, Pittsburgh, Pennsylvania, USA 
Monday

0800-1630

$\mathbf{P ( 4 7 ) ( 3 5 . 8 ) \times x 7 4 . ~}$

Japan's International Disaster Relief

Seki $T$

Disaster Relief Division, Medical Cooperation Department, Japan

International Cooperation Agency, Tokyo, Japan

P(48)(35)(40.3)xxx33.

Vibration-Proof Construction Engineering

for Medical Institution within Earthquake Zone

Teramura A, Yomaru $K$

Obayashi Corporation, Technical Research Institute, Tokyo, Japan

$\mathbf{P ( 1 ) ( 3 4 . 2 ) \times x x 5 7 .}$

Disaster Medicine in the Netherlands

De Boer J

Civil Defence and Peacetime Calamities, Ministry of Welfare, Health, and Culture, Rijswijk, Netherlands

\section{$\mathbf{P}(2)(34.5) \times x \times 68$.}

Innovations in California Disaster Medical Preparedness

Freeman $C$

California EMS Authority, Sacramento, California, USA

California faces a variety of serious disaster threats including floods, volcano eruptions, and earthquakes. Man-made threats include release of hazardous materials, mentally deranged gunmen, fires, and airplane crashes. The severity of these threats and the frequency of disasters within the state have prompted California's state, county and local governments, hospitals, and medical responders to develop innovative approaches to medical preparedness and response problems. This poster session will summarize some of the more significant activities that have been developed. These activities include: 1) Computer simulation of CCP operations; 2) Application of the Incident Command System to the organization of hospital disaster response; 3) Statewide standards for multiple casualty incident planning and response; 4) Unique joint military-civilian operations to direct the state's medical response; and 5) Hospital earthquake rapid assessment and reporting.

$P(3)(35.18) \times x 160$. Poster and Video Presentation Major Bus Accidents In Rural New South Wales, Australia Jacobson J, Juul O, Selig $M$ Ambulance Education Centre, Royal North Shore Hospital, St. Leonards, Australia
Tuesday, May 14, 1991

0800-1630

$\mathbf{P}(4)(30)(31.0) \times x \times 52$.

Telefacsimile Could Be the Solution

to Information Breaks at the Scene

Mattila MAK, Koski EMJ

Kuopio University Hospital, Kuopio, Finland

As seen in the large-scale disaster exercises, the problem of establishing and maintaining proper communication systems during disaster situations is demanding. In spite of numerous walkie-talkie and cellular telephones available, very little documentation can be recorded of the verbal telecommunications between different groups and persons. Therefore, the use of telefax is proposed to improve the efficacy of providing information during emergencies.

The superiority of telefax to telephone stems from its ability to recover documents, which are more durable and less transient than verbal instructions, both during the emergency and as part of the post-emergency reconstruction. For example, telefax permits the transmission of updated maps that highlight dangerous areas, traffic limitations, and positions of important groups in a changing situation. Lists of persons rescued also can be transmitted faster and more reliably than if spoken by telephone. Thus, the use of the telefax is more versatile, complete, and illustrative than is verbal information. It frees up more lines for communication, and in some telefax apparatus, the message also can be processed by computer.

These excellent devices are portable, light, and applicable to a variety of field situations, particularly with the use of cellular telephones. The development of a more durable and portable machine would make it even more useful in the field and would permit base station/command center communications with emergency, on-scene personnel. As there would be several different locations and telephone numbers for telefax machines in the field, there also would be a corresponding need for several telefax machines at the command center.

At present, this proposal is theoretical and requires critical evaluation and assessment during demanding exercises. 
Tuesday

0800-1630

$\mathbf{P ( 5 ) ( 3 . 2 ) \times x 7 9 . ~}$

Personality Profiles of EMS Providers:

The Development of an EMS Assessment Centre

for Selection, Promotion, and Career Counseling

Williams AT

Emergency Health Services Academy, Justice Institute of British

Columbia, Vancouver, British Columbia, Canada

In 1989, the British Columbia Ambulance Service (BCAS) introduced a personality profiling exercise as a team-building exercise for its managers. In 1990, it developed a personality profile common to basic EMS providers, and in 1991, a profile was developed for its Advanced Life Support (paramedic) providers. Matching candidates' personality profiles against a required job profile is a useful component of recruitment, advancement, and supervisory selection. These profiles also can be used for career counseling for problem employees and as a part of a larger career development process. The success of the project provided the impetus to develop a more comprehensive assessment centre process specifically for BCAS, a large, provincial EMS system.

This presentation will describe the personality profile of the EMS provider, and how the assessment tool can be used for candidate selection, promotion, and career counseling. It also will describe the job dimensions of BCAS paramedics, and how they were developed. Finally, it will discuss the advantages and disadvantages of the Assessment Centre method.

$\mathbf{P ( 6 ) ( 3 5 . 1 5 ) \times x 1 2 3 . ~}$

A New EMS Incident Command System

Rega $P, M c$ Connaughy D, Mack D

The Toledo Hospital, Toledo, Ohio, USA

$\mathbf{P}(7)(29.3) \times x \times 178$

Use of Serum-Acupuncture in Acute

and Chronic Destructive Diseases

Rosenberger FM

International Pain Relief Association, Graz, Austria
Tuesday

$0800-1630$

$\mathrm{P}(8)(19.5) \times x x 21$.

Inter-disciplinary Fellowship Program

in Disaster Reanimatology for Physicians

Safar P, Pretto E, Angus D, Ricci E, Klain M, Comfort L, Paris P, Abrams J

International Resuscitation Research Center (IRRC), and Schools of Medicine, Public, and International Affairs, and Engineering,

University of Pittsburgh, Pittsburgh, Pennsylvania, USA

In the past, disaster research was conducted mostly in public health and sociology. At the founding of the Club of Mainz (WAEDM) in 1976, the author proposed studies by reanimatologists of resuscitation potentials in mass disasters. Retrospective studies after earthquakes in Peru and Italy resulted in a study in Armenia [USSR] by a multi-disciplinary team. The study indicated that disaster reanimatology needs medical leaders with inter-disciplinary backgrounds for research, planning, and response. A program for interested physicians experienced in emergency, critical care medicine, or traumatology was initiated.

The one-year program includes courses in public health concerning epidemiology, computer science, statistics, and EMS evaluation (Ricci); and in international disaster management (Comfort). If lacking adequate levels of training and experience, the Fellow is provided with prehospital clinical experience and certification in ACLS, ATLS, and base station medical command (Paris). The curriculum includes participation in local EMS council meetings (Paris), "resuscitative engineering" (Abrams), visits with national and international leaders in disaster medicine, and guided literature research. There are weekly seminars with the program directors (Safar, Pretto) and monthly disaster seminars with faculty. The Fellow also interacts with sociologists via a multi-professional, university-wide, disaster research committee. Finally, the Fellow is guided in the writing of a review paper, and in updating of field study protocols and "instruments" (Klain, Ricci). If possible, the Fellow also participates as a member of a multi-professional team in a field study soon after the occurrence of a disaster. Experience with this program will be presented.

$P(9)(6.8) \times x x 116$.

Kinetic Analysis on the Recovery of Cholinesterase

After Cholinesterase Inhibitor Poisoning in Humans

Shirakawa Y, Tsukamoto I, Seki K, Aibiki M, Ohli K

Department of Anesthesiology and Emergency Medicine, Kagawa

Medical School, Kagawa, Japan 
Tuesday

$0800-1630$

P(10)(20-1) $\times x \times 41$.

Tympanic Membrane

and Rectal Temperature in Marathon Runners

Silverman, Grant SAD, Rennie WP

Department of Emergency Medicine, Long Island Jewish Hospital, New Hyde Partk, New York, USA

Introduction: Endurance Athletes are at risk for both heat stroke and hypothermia. Rectal Temperatures (RT) are the standard for estimating core temperature but are difficult to obtain in the prehospital setting. The study purpose was to determine if tympanic membrane temperatures (TMT) correlated with RT in Marathon runners in the prehospital setting.

Materials and Methods: Runners presenting to the medical tents at the 1989 and 1990 New York City Marathon had RT determined with an electronic thermometer (TEMP-PLUS II, IVAC Corp., San Diego, Calif.). At the same time, TMT were determined in the right and left ears of each patient using one of two different infrared ear thermometers (FirstTEMP, IMS Inc., Carlsbad, Calif., or EXERGEN 3000, Exergen Corp., Newton, Mass.). Results were analyzed using Pearson correlation coefficients and paired T-tests.

Results: (Units $={ }^{\circ} \mathrm{C} \pm 2 \mathrm{SE}$ ) There was high correlation between the right and left ear TMT for each instrument (EXERGEN, Pearson r2=0.923; FirstTEMP, Pearson r2=.0871). However, there were significant differences found between TMT and RT. The FirstTEMP TMT mean was $36.53 \pm 0.29$ compared with a mean RT of $37.84 \pm 0.38(\mathrm{n}=48, \mathrm{p}<.0001)$. The Exergen TMT mean was $36.90 \pm 0.56$ compared with a mean RT of $38.51 \pm 0.64$ $(\mathrm{n}=26, \mathrm{p}<.0001)$. Notably, three runners with heat stroke has mean RT of $42.1^{\circ} \mathrm{C}$ and mean TMT of $39.5^{\circ} \mathrm{C}$. Overall, the correlation between TMT and RT was relatively low. (Exergen, Pearson r2=0.686; FirstTemp, Pearson r2=0.686).

Conclusion: Tympanic membrane temperatures derived by two different infrared thermometers differ significantly from rectal temperatures in marathon runners in the prehospital setting.

\section{Tuesday}

0800-1630

P(11)(23.4)xx130.

Outcome in Patients with Traumatic Intracranial

Hemorrhage Related to Time of Operation

Takasu A, Taneda M, Otuki H, Okamoto H, Masana Y, Morishita $Y$

Department of Neurosurgery, Hanwa Memorial Hospital, Osaka,

Japan

Introduction and Methods: In order to investigate the timing of emergency operation on morbidity and mortality in head injury, 24 patients with intracranial mass hemorrhagic lesions undergoing an craniotomy within 24 hours after injury were selected for this study. They were classified into two groups: 15 patients were operated-on within six hours after injury (Group A); and 9 patients were operated more than six hours after injury (Group B).

Results: There were no remarkable differences between two groups in relation to age, type and location of hemotoma, or level of consciousness within one hour after injury. Most of the patients in both groups deteriorated before the operation. Ten patients $(67 \%)$ of Group A showed good recoveries in terms of Glasgow Outcome Scale score, whereas only one patient in Group B (11\%) achieved good recovery.

Conclusions: This study concludes from these results that the timing of operation has a crucial influence on the outcome of head injured patients who have an intracranial hemorrhagic lesion. Finally, the causes of delays of operation will be discussed not only from a medical, but also a social perspective.

$\mathbf{P}(12)(6.7) \times x \times 42$.

Health Risk Assessment for Major Chemical Hazards: The Use of Exposure Effect/Response Relationships for Safety Promotion in General and for Disaster Response van der Torn $P$ Municipal Health Service of Rotterdam Area 
Wednesday, May 15, 1991

$0800-1630$

$\mathrm{P}(1)(14.1) \times \times 91$.

The Influence of Postischemic Reperfusion Injury on the Kidney Following Suprarenal Aorta or Renal Artery Clamping

Tsuchiya A, Wakabayashi Y, Masaki Y, Ohwada T

Department of Emergency and Critical Care Medicine, Kitasato

University School of Medicine, Sagamihara, Japan

$P(2)(34.11) \times x \times 43$.

Evaluation of the Continuum

of Health Effects for Safety Promotion

van der Torn $P$

Municipal Health Service of Rotterdam Area

Safety promotion is divided globally into four phases: 1) prevention; 2) preparation; 3) repression (disaster response); and 4) aftercare. The perspective on the continuum of health effects varies by the phase of safety promotion.

An integrated view on both short- and long-term health effects is needed for the prevention of and preparation for disasters, as well as for some aspects of disaster response (protection of the population in a threatened area). Sets of effect-levels, such as those proposed by the American Industrial Hygienists Association (AIHA) and the European Chemical Industry Ecology and Toxicology Centre (ECETOC), serve these purposes reasonably well. Basically, the 3-D or 4-D effect levels are distinguished: death; disability; discomfort; and detectability.

Disaster response in the medical chain of assistance requires not so much a description of the continuum of (short-term) health effects as such, but is helped more with a definition of intervention levels. The Dutch uniform triage system for mechanical lesions is thought to form a good point of departure for defining a set of intervention levels for toxic lesions. Such a triage-system will be proposed and some characteristics with be presented.

Aftercare necessitates an altogether different viewpoint on the continuum of health effects. Only long-term health effects are at issue, and much more attention is paid to psychosocial health effects (e.g., related to the post-traumatic stress syndrome) than could be the case with disaster response. The data base for long-term health effects seems sufficiently well-established to strive for sets of effect levels by target organ. Some examples will be presented.
Wednesday

$0800-1630$

$\mathbf{P}(3)(38.3) \times \times 77$.

A Multi-Disciplinary First Responder Program:

Providing a Continuum of Prehospital Care

Williams AT

Emergency Health Services Authority, Justice Institute of British

Columbia, Vancouver, British Columbia, Canada

Many different agencies provide first aid or prehospital care. In British Columbia (BC), the primary first responders, police, fire, and ambulance paramedics were trained to assess and treat victims differently. This led to confusion and some on-scene frustrations among the first responders. In 1989, the Chief Coroner of $B C$ reviewed the situation and recommended that a unified approach be adopted by the professional first responders. The Justice Institute developed such a program and tailored it to the needs of the three services.

It has been an unqualified success. All three services are following a medical model that ensures a continuum of care into the emergency departments.

This presentation will describe the contents of each of the four levels of the program. It will describe the student evaluation method and how the provincial standard is maintained. It also will describe the process by which police, firefighters, and paramedics came together to develop the program and its implications for future cooperative programs.

P(4)(27-1) $x \times x 34$.

Septic Complication and DIC in Extensive Burn Patients Kurokawa A, Makino T, Susaki SI, Henmi HH, Otsuka T, Yamamoto $Y$

Department of Emergency and Critical Care Medicine, Nippon Medical School, Tokyo, Japan 
Wednesday

0800-1630

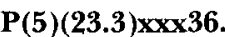

Significance of Magnetic Resonance Imaging

(MRI) in Evaluation of Diffuse Axonal Injury

Yokota H, Henmi HH, Yamamoto YY, Ostuka TO

Department of Emergency and Critical Care Medicine, Nippon

Medical School, Tokyo, Japan

The evaluation of patients with head injuries has improved significantly since the introduction of computerized tomography (CT). But CT has failed to demonstrate small lesions, such as white matter or brainstem defects. Magnetic resonance imaging (MRI) is thought to be superior to CT for visualizing these lesions. However, the usefulness of MRI in acute head injury, especially in axonal injury, has not been explored fully. This paper discusses the usefulness of MRI compared with imaging by CT in detecting diffuse axonal injury.

Over the past two years, 232 patients with head injuries underwent studies with MRI within three days of their injury. Of these, 26 patients were diagnosed as having diffuse axonal injury according to the criteria established by Gennarelli. Each patient received CT within three hours following the first MRI study. The advantages of MRI to CT in the evaluation of diffuse axonal injury (DAI) are discussed.

These findings suggest that MRI is able to demonstrate the lesions as a high intensity area on T2-weighted imaging in mild and moderate DAI. These lesions, which are not demonstrated on CT, but are demonstrated on MRI, are considered non-hemorrhagic contusions. The main lesions in mild or more moderate DAI are located in cerebral white matter, and the lesions in severe DAI are located in the brainstem, subependymal layer, and/or corpus callosum, as well as the cerebral white matter.

This study suggests that MRI is more sensitive than CT in detecting the lesions of diffuse axonal injury.

\section{$P(6)(06) \times x \times 190$.}

\section{Effect of BAYK8644 in Acute}

Verapamil Intoxification in the Dog

L'Heureux $P$, Vranckx $M$, Leduc D, Askenasi $R$

Emergency Department, Clinical Toxicology, Erasme University

Hospital, Brussels, Belgium

The drug BAYK8644 is a dihydropyridine compound with calcium entry promoting activity. the purpose of this study is to evaluate its antidotal value against cardiovascular disturbances produced by acute experimental verapamil (VP) intoxication in the dog model.

Fifteen dogs were anesthetized with sodium pentobarbital, curarized, and ventilated. Heart rate, femoral arterial, right cardiac, and pulmonary pressures are continuously recorded. Cardiac output (thermodilution) and arterial $\mathrm{pH}$ are serially determined. An infusion of VP $(0.15 \mathrm{mg} / \mathrm{kg}-1 / \mathrm{min}-1)$ is administered during $30 \mathrm{~min}$ (T0-T30) in all the animals. It is associated to an infusion of BAYK8644 (5 $\mu \mathrm{g} / \mathrm{kg}-1 \mathrm{~min}-1)$ from T0 to $\mathrm{T} 30$ in four dogs and from T15 to T45 in four others.

The drug BAYK8644 does neither prevent nor correct the bradycardia and the conduction disturbances produced by VP. Conversely, it antagonizes the drop of arterial pressure. this effect results mainly from peripheral vasoconstriction and, in a lesser extent, from a slight improvement in myocardial acidosis is not prevented. These results suggest a limited antidotal value of BAYK8644 in VP intoxication.
Wednesday

$0800-1630$

$\mathbf{P}(7) \times x \times 190$.

The Role of the Public Health Service

in Disaster Medicine

Costanzo $S$

Department of Public Health, Rome, Italy

$\mathbf{P}(\mathbf{8})(\mathbf{3 4 . 3}) \times \mathbf{x x 5 8}$.

The Definition and Classification of Disasters

De Boer J

Director, Civil Defence and Peacetime Calamities, Ministry of Welfare, Health, and Culture, Rijswijk, Netherlands

Any disaster, either man-made or natural, can produce a very large number of casualties in a short period of time, often at a time when the community is least capable to come to their aid. Disaster Medicine is that part of the art and science of medicine which studies the clinical and organizational problems of disasters. It is a young branch of medicine, and it still provokes confusion because people use similar terms in different ways. The foundation of science is definition, classification, and measurement, and if Disaster Medicine is to grow, it also must have its definitions, classifications, and measurements. The concept of definition, as well as the methodology for classification and scoring is presented.

$P(9)(29.2) \times x \times 177$.

Acupuncture in the Treatment of Accident Victims

Rosenberger $F M$

International Pain Relief Association, Graz, Austria

$P(10)(19-6) \times x 94$.

Postgraduate Emergency Medical Training in Japan

Ukai T

Osaka Prefectural Senri Critical Care Medical Center, Osaka, Japan 